\title{
Using Mobile 3D Scanning Systems for Objective Evaluation of Form, Volume, and Symmetry in Plastic Surgery: Intraoperative Scanning and Lymphedema Assessment
}

\author{
Konstantin Christoph KOBAN, Riccardo Enzo GIUNTA \\ Department of Hand Surgery, Plastic Surgery and Aesthetic Surgery, \\ Ludwig-Maximilians-University, Munich, Germany
}

\begin{abstract}
\section{Background:}

There has been ongoing development in the field of three-dimensional (3-D) Surface Imaging such as laser scanner and digital photogrammetry in recent years. Manufacturers tend to make new 3-D cameras compact, light-weighted, mobile, and user-friendly, similar to the development on the smartphone market.
\end{abstract}

Although 3-D scans have been used for patient consultations and digital documentation in Plastic Surgery since the 1980ties, there has been no significant development lately regarding its use for objective assistance during surgery.

\section{Patients, Material and Methods:}

Our research team presents the use of different mobile scanning systems (Sense, iSense, Artec Eva, and Thor) as a new intraoperative 3-D scanning method for plastic-surgical procedures. We present several cases of aesthetic breast shaping and breast reconstructions with implants and free tissue transfer, such as lipofilling and complete reconstructions with microsurgical free flaps. In the future, these might assist surgeons with the pre-, post-, and intraoperative 3-D analysis, choice of therapy, consultation, and documentation.

Furthermore, we want to present the same scanners for the accurate assessment of arm and leg lymphedema patients. The diagnosis of lymphedema in patients and especially the outcome after treatment has been described with various methods so far. But in terms of clinical practicability physicians still rely on classical tape measurement. We compare volume estimation between the results of our 3D scanners against tape measurement and water-displacement.

\section{Results:}

The 3-D scanners were successfully validated for their intraoperative application to several patients. An intraoperative, objective measurement of volume to evaluate form and symmetry was possible for aesthetic procedures, such as breast augmentation and reduction, as well as for breast reconstruction with lipofilling and free tissue transfer. 3D scanning was used for key steps in all these procedures with minimum delay of the procedures and instant feedback for the surgeons. There was nonetheless significant difference between the used 3-D scanners in terms of mesh quality and textures.

We could also validate all mobile scanners as objective tools to capture lymphedema in patients for documentation before and after treatment. The biggest difference was shown against tape measurement, which is highly dependent on correct placement and the different estimation methods.

\section{Conclusion:}

In this work, we share our first experience with the intraoperative use of new mobile 3-D camera systems, discuss pros and cons, and show selected patient examples. The newest mobile scanning systems showed highly accurate 3-D scans during surgery regarding the actual form, shape, volume shifts and differences.

Furthermore, mobile 3-D camera systems were able to accurately assess lymphedema especially for the leg and may become the future chosen method to evaluate different treatments of lymphedema.

Although the development of 3-D scanning devices rapidly increased, we still lack appropriate software development for medical usage. Newer software has to become more practicable, userfriendly, and especially versatile for different plastic surgical procedures. 

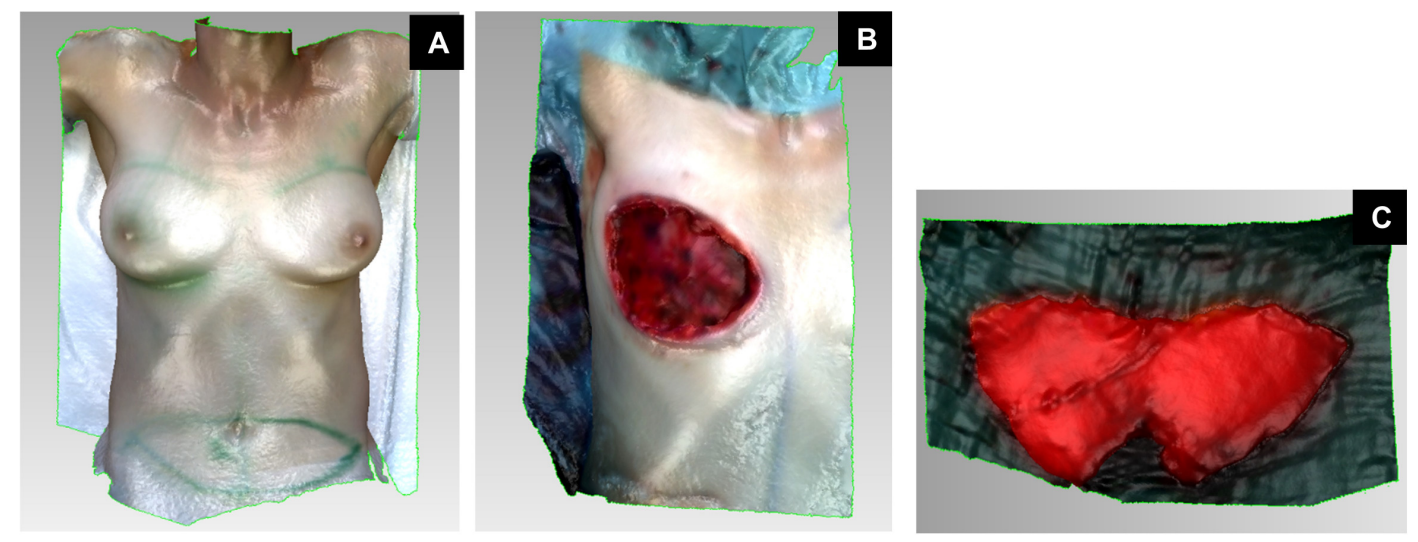

Fig. 1: Intraoperative Scan (A: before cancer resection, B: after complete ablation, C: the free DIEP flap) before breast reconstruction with the Sense Scanner.
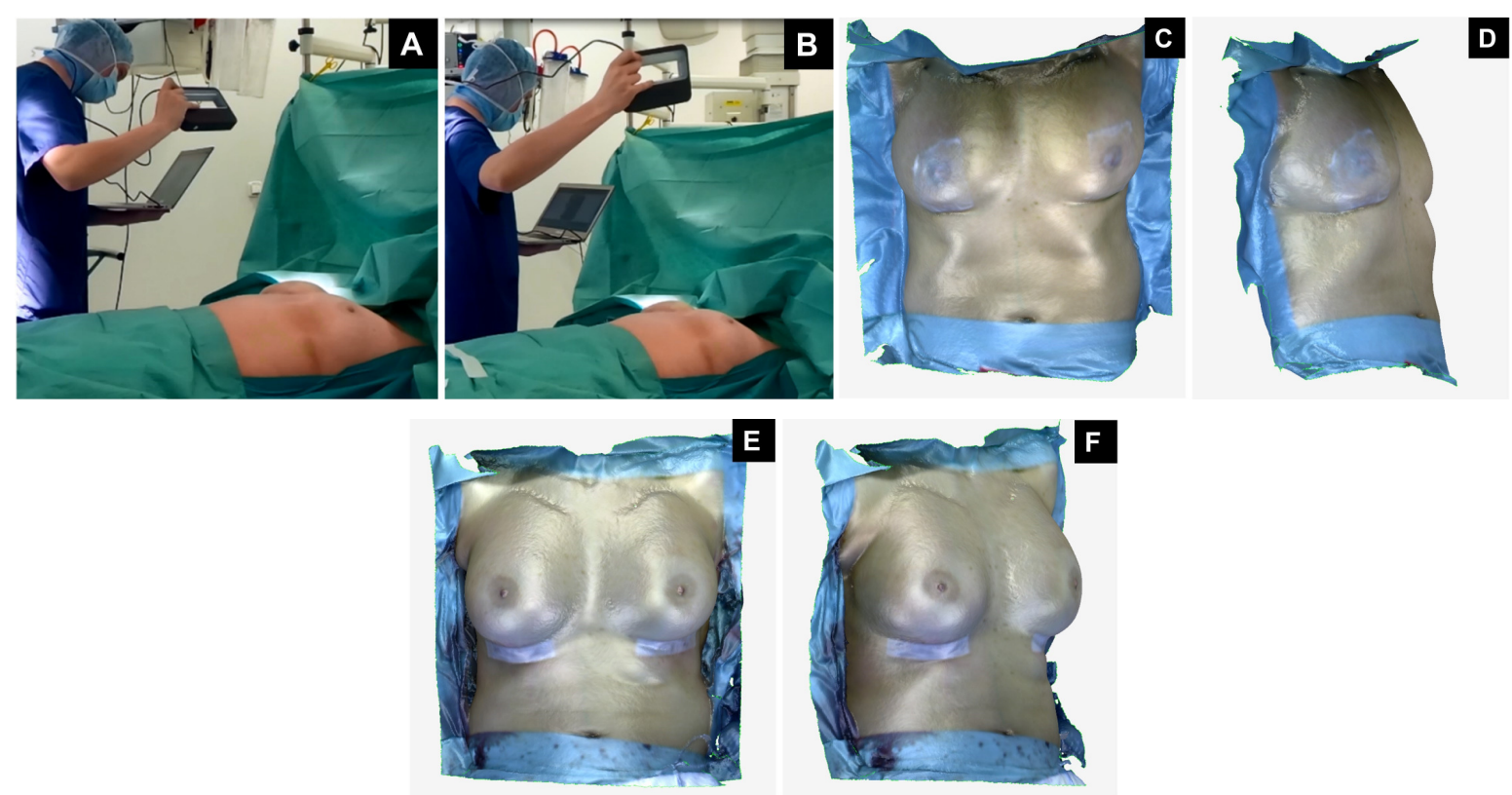

Fig. 2: Intraoperative 3-D Scan with the Sense in an aesthetic breast augmentation. $A-B$ shows the scan method, $C-D$ the preoperative, and $E-F$ the postoperative results intraoperatively.
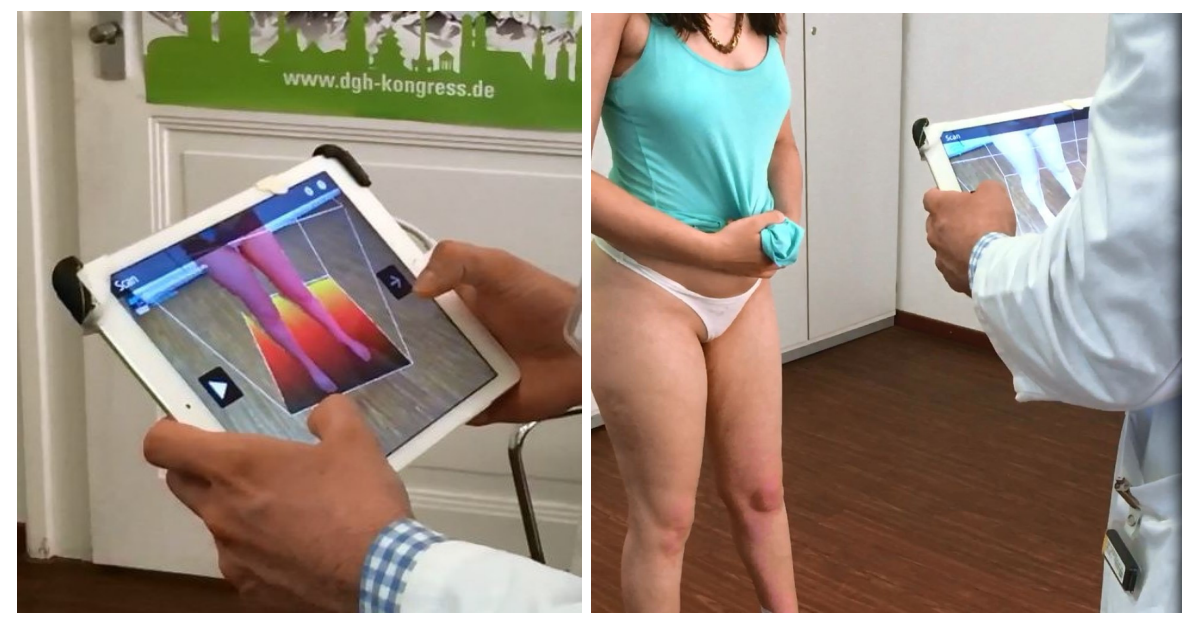

Fig.3: 3-D Scan with the iSense of an young female with lymphedema of her thighs. 